\title{
Psychiatric Comorbidities and the Risk of Suicide in Obsessive-Compulsive and Body Dysmorphic Disorder
}

\author{
Noha Eskander ${ }^{1}$, Therese Limbana ${ }^{1}$, Farah Khan ${ }^{1}$ \\ 1. Psychiatry, California Institute of Behavioral Neurosciences \& Psychology, Fairfield, USA
}

Corresponding author: Noha Eskander, eskandernoha@gmail.com

\begin{abstract}
Obsessive-compulsive disorder (OCD) is a chronic psychiatric disorder that is characterized by obsessions and compulsions. Obsessions are uncontrollable distressful thoughts. Compulsions are recurrent behaviors or thoughts performed in an attempt to decrease the anxiety of the obsessions. Body dysmorphic disorder (BDD) is a mental disorder characterized by a distressful preoccupation with a perceived defect in appearance. The perceived flaw in appearance is minimal or unnoticed by others. BDD was considered an anxiety disorder in the Diagnostic and Statistical Manual of Mental Disorders, fourth edition (DSM-IV). In the DSM-V, it was added to the obsessive-compulsive and related disorders category. The objective of this literature review was to explore the psychiatric comorbidities and the risk of suicide associated with OCD and BDD. Our study results showed OCD and BDD share common genetic and environmental risk factors, clinical features, and sociodemographic profiles. Both OCD and BDD are related disorders that commonly coexist. The suicide risk in OCD is increased as the intensity of the obsessions, trait perfectionism, and alexithymia increases. The suicide risk in BDD is increased by the presence of other disorders such as substance use disorder, major depressive disorder, eating and personality disorders. People with comorbid OCD-BDD have high morbidity, a decrease in insight and poor psychosocial functions. They have higher rates of anxiety, schizotypal features, and suicidal ideation compared to those with BDD or OCD alone.
\end{abstract}

Categories: Neurology, Psychiatry, Psychology

Keywords: public psychiatry

\section{Introduction And Background}

Obsessive-compulsive disorder (OCD) is a chronic mental disorder. It is characterized by obsessions and compulsions. Obsessions are uncontrollable recurring and distressful thoughts. Compulsions are repetitive behaviors which the person feels the urge to perform in an attempt to decrease the anxiety of the obsessions. The estimated prevalence of OCD among adults in the United States for a 12 -month period is $1.2 \%$ and for a lifetime is $2.3 \%$. The mean age of OCD onset is 19.5 years [1]. There is a significant gender difference in the OCD age of onset. Males usually start developing symptoms before the age of 10 compared to females who usually start to develop symptoms after the age of 10. Most individuals with OCD spend a mean of 8.9 years of their life with the disorder [1].

Received 08/02/2020 Review ended 08/16/2020 Published 08/17/2020

\section{() Copyright 2020}

Eskander et al. This is an open access article distributed under the terms of the Creative Commons Attribution License CC-BY 4.0., which permits unrestricted use, distribution, and reproduction in any medium, provided the original author and source are credited.
Body dysmorphic disorder (BDD) is also known as dysmorphophobia. BDD was considered an anxiety disorder in the Diagnostic and Statistical Manual of Mental Disorders, fourth edition (DSM-IV). But in the DSM-V was added to the obsessive-compulsive and related disorders category. BDD is a psychiatric disorder characterized by an excessive, persistent, and distressful preoccupation with a perceived defect in appearance. These perceived defects are slight and are unnoticed by others. People with BDD usually have poor insight and are preoccupied with a perceived physical defect which causes them to check on it repeatedly. This leads to an impairment in psychosocial functioning, depression, and an increase in suicide risk [2].

The prevalence of BDD in the adult community is $1.9 \%, 7.4 \%$ in psychiatric inpatients, $13.2 \%$ in general cosmetic surgery, $20.1 \%$ in rhinoplastic surgery, and $9.2 \%$ in cosmetic outpatient clinics [3]. BDD is an underdiagnosed disorder. Many people suffering from BDD do not seek psychiatric advice, instead, they are usually diagnosed in cosmetic clinics and other settings because they do not recognize they have a problem. Others prefer not to disclose their condition because they are afraid to be judged or because they feel embarrassed [4]. BDD is usually diagnosed in adolescence [5]. It is more common in females than males with a prevalence of $2.1 \%$ and $1.6 \%$, respectively [2].

Historically, the risk of suicide in patients with OCD was considered low [6]. Recent studies found about 63\% of individuals with OCD had suicidal thoughts and $26 \%$ had suicidal attempts [7]. The presence of comorbid disorders significantly increases suicide risk [7]. People with BDD are four times more likely to experience suicidal ideation and $2.6 \%$ more likely to attempt suicide than individuals without BDD [8]. 
The objective of this literature review was to explore what psychiatric comorbidities are associated with OCD and BDD and to review the risk of suicide in these two disorders. The study review also aimed to understand the risk factors, clinical differences, comorbidities, and suicide risk in comorbid OCD and BDD.

\section{Review}

\section{Methods and results}

Data were searched on Pub Med using regular keywords "Obsessive-Compulsive Disorder", "Body Dysmorphic Disorder". Table 1 shows the search results of the regular keywords "Obsessive-Compulsive Disorder”, "Body Dysmorphic Disorder".

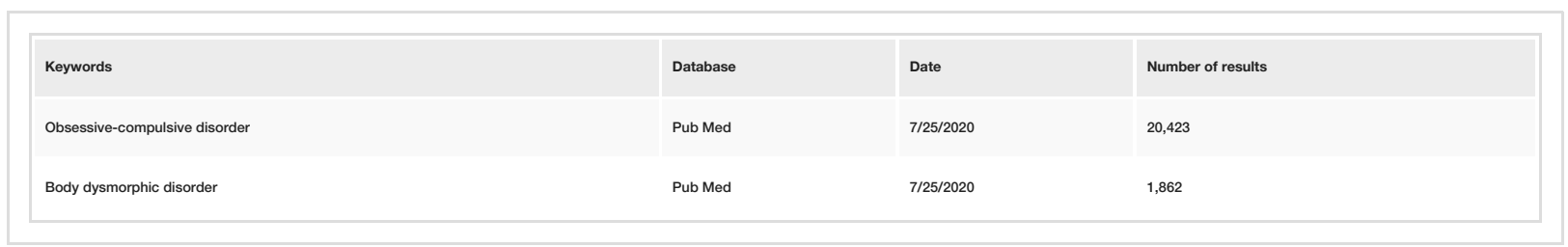

TABLE 1: PubMed keywords search results

\section{Results}

The total number of keywords search results was 22,285 . The following is a breakdown of the keywords searched and the volume of results: Obsessive-Compulsive Disorder keyword search results were 20,423. Body Dysmorphic Disorder keyword search results were 1,862 .

Only research articles related to human studies that were published since 1998 in the English language were included in this study. All types of research articles were included except for books and documents. After the manual screening of each article, the relevant research studies for this literature review were selected. A total of 31 articles were selected for this study to determine the psychiatric comorbidities and the risk of suicide in obsessive-compulsive and body dysmorphic disorder.

\section{Discussion}

The results of the study showed that both OCD and BDD are associated with poor psychosocial outcomes, an increase in the associated comorbidities, and suicidal risk. Individuals with comorbid OCD and BDD are at an increased risk of depression, anxiety, and suicide.

Obsessive-Compulsive Disorder

OCD is a common mental disorder that is often under-diagnosed. Patients with OCD might wait for years before they seek medical help or receive the right diagnosis. This is due to a lack of awareness and stigma around OCD. Symptoms of OCD include obsessions such as fear of contamination, doubts about harm, unacceptable thoughts and concerns about symmetry, and their respective compulsions in response to their obsessions such as cleaning, repeatedly checking on things, mental rituals, and placing items in order [9]. About 25\% of OCD cases reported obsessions that are not associated with compulsions [10]. Neuroimaging studies showed that OCD is associated with an increase in the activity in a brain circuit that involves the orbitofrontal cortex (OFC), striatum, and thalamus. These findings might reflect glutamatergic dysfunction in OFC-striatal pathways. OCD is also associated with Serotonergic dysfunction based on the efficacy of SSRI (selective serotonin reuptake inhibitors) in treating OCD [11]. Genetic studies showed OCD is a heritable disorder that runs in certain families with $45 \%$ to $65 \%$ genetic contribution [12].

Approximately $90 \%$ of individuals with OCD have other psychiatric comorbidities. The most common comorbid disorder in OCD is anxiety disorders with a prevalence of $75.8 \%$, mood disorders with $63.3 \%$ specifically major depression disorder (MDD) with 40.7\%, impulse control disorders $55.9 \%$; and substance use disorders (SUDs) $38.6 \%$ [7]. ADHD, tic disorders, and hypochondriasis are also common with OCD [13]. The comorbidity of OCD and depression significantly increases the risk of suicide [7]. It is believed that OCD symptoms predate depression due to the distressful and the functional impairment experienced by people with OCD. Depression is more associated with obsessions than compulsions, the higher the frequency and intensity of the obsessions, the higher the number of suicide attempts [14]. A systematic review by Albert et al. found the severity of comorbid anxiety symptoms in OCD patients is associated with a high risk of suicide [15]. Additionally, comorbid bipolar disorder (BD), cigarette smoking, and a history of abuse all predict higher suicidal risk in patients with OCD [15].

A study by Kim et al. $(n=81)$ found that trait perfectionism is associated with high suicidal risk [16]. A crosssectional study $(n=548)$ found the increase in the suicidal continuum is associated with an increase in 
aggression, religious and sexual thoughts, and concerns about symmetry and order [17]. Other factors that were associated with suicide were previous suicide attempts and a family history of suicide [17]. A cohort study $(n=36,788)$ found that OCD patients are ten times more likely to commit suicide than the general population [6]. Women are more likely to attempt suicide than men. The most method used to commit suicide in OCD patients was self-poisoning possibly due to the availability of psychotropic drugs. Comorbid personality disorder and substance use disorders were also associated with a higher risk of suicide [6]. A case report study described a case of OCD with suicidal obsessions [10]. The study emphasized the importance to differentiate suicidal ideation from suicidal obsessions. Suicidal obsessions are stressful, repetitive, and intrusive thoughts that are highly undesirable and avoidable. Suicidal obsessions are not associated with a desire to act on them. Suicidal ideations are thoughts about self-harm with serious intent to act on them and they are not necessarily intrusive or stressful. It is important to properly investigate patients presenting with OCD and suicidal ideation to avoid confusion with suicidal obsessions [10].

Table 2 summarizes some of the important studies that explain the risk of suicide in patients with OCD.

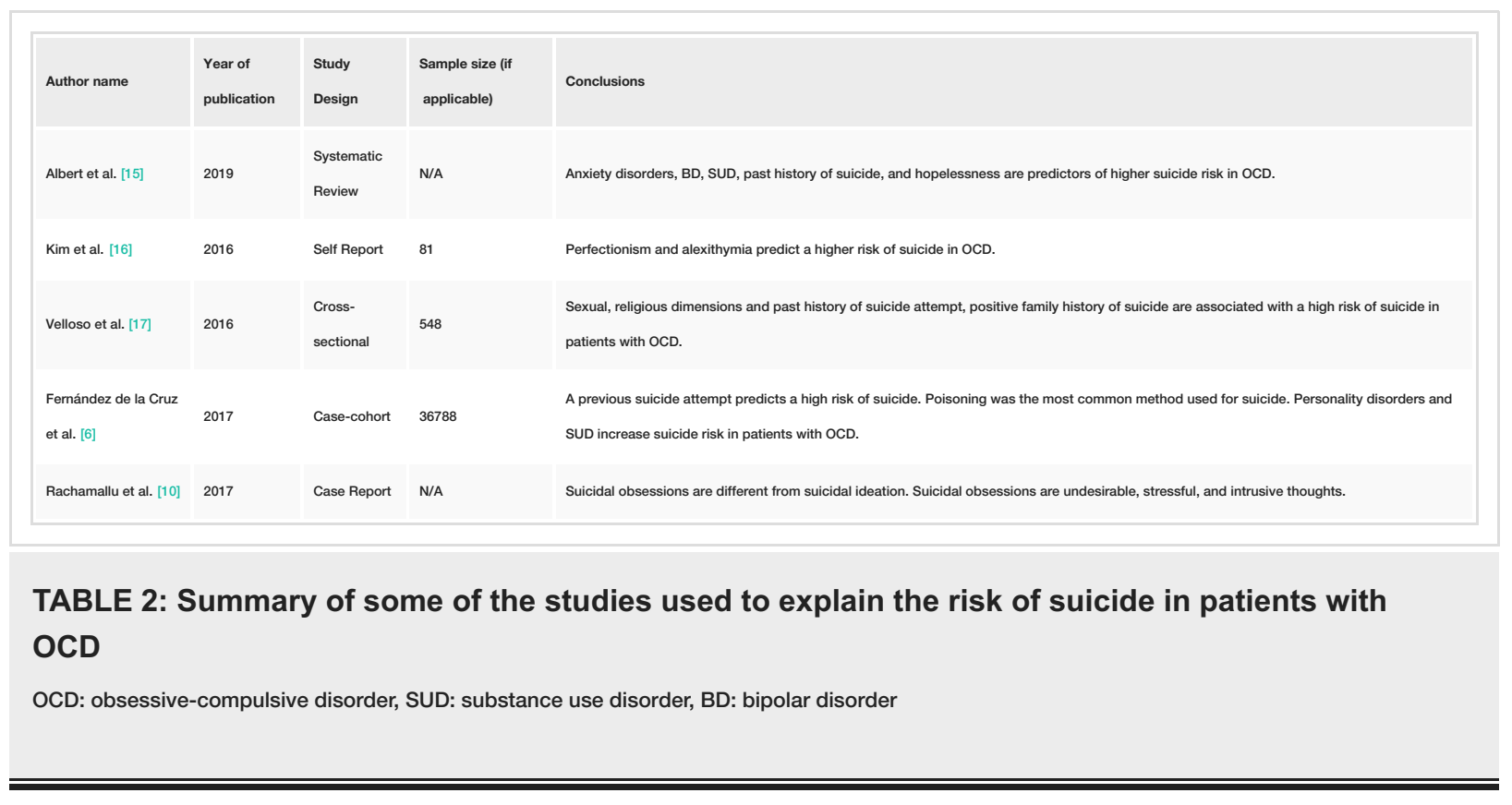

Body Dysmorphic Disorder

People with BDD are overly preoccupied with one or multiple features in their body which they believe look flawed. They repetitively and compulsively check the perceived defect in front of a mirror or ask others about their appearance. The preoccupation is obsessive and consumes many hours per day. Studies of twins showed that approximately $43 \%$ of genetic factors contribute to BDD symptoms. Bullying, childhood abuse, peer teasing are risk factors for the development of BDD [18]. Patients with BDD are often presented with anxiety and depression so their symptoms are commonly misdiagnosed as social phobia, MDD, or OCD [19]. Eating disorders like bulimia and anorexia nervosa are common disorders in patients with BDD [20]. BDD is present in about $25 \%$ to $39 \%$ of patients diagnosed with anorexia nervosa. Those patients have a high rate of hospitalization and a three times increase in suicide attempts [21]. Muscle dysmorphia is a form of BDD that is common among men. Men with muscle dysmorphia believe they are not muscular enough when in fact they might look unusually muscular. They may take anabolic steroids, follow a strict diet, and adhere to time-consuming work-out schedules. They might avoid social events and occupational activities because of the shame on their perceived appearance. Men with muscle dysmorphia have a poorer quality of life, an increase in substance use and suicide risk [22].

People with BDD often feel depressed. Studies found $24 \%$ to $28 \%$ of individuals diagnosed with BDD attempt suicide. Suicidal ideation and attempts are associated with an increase in symptom severity and marked psychosocial dysfunction. Risk factors for suicide ideation and attempts in BDD include comorbid BD, SUD, MDD, post-traumatic stress disorder, personality disorders especially borderline personality disorder [23]. An observational study $(n=200)$ found $94.6 \%$ of adolescents with BDD have reported distress and severe impairment socially, academically, and psychologically and $44.4 \%$ had suicide attempts [24].

Comorbid $O C D$ and $B D D$

In the DSM-V, BDD was added to the obsessive-compulsive and related disorders category together with OCD, hoarding disorder, trichotillomania, and excoriation disorder. Both OCD and BDD share common features such as: 
1. Genetics and environmental influences: past studies found high rates of both disorders among first degree relatives of patients with either OCD or BDD. They also found a high prevalence of other mental health disorders such as social phobia, depression, and eating disorders in these families [25]. A genetic study ( $\mathrm{n}=$ 2,148 ) of adult twins found the common traits in OCD and BDD are due to genetic overlap of $64 \%$ in these two disorders [26]. Regarding the environmental factors, a cross-sectional study $(\mathrm{n}=100)$ found significantly higher rates of emotional and sexual abuse in BDD samples as compared to OCD samples. Both groups reported the same rates of physical abuse [26].

2. Socio-demographic profile: both disorders have very similar gender ratios and close social profiles with the exception of BDD which is slightly more common among single patients [27].

3. Psychopathological symptoms: people with BDD spend long hours performing ritualistic behaviors such as spending hours grooming, checking, and obsessing about their appearance. These repetitive behaviors are similar to compulsions in patients with OCD. People with BDD have less insight about their disorder and are more likely to be delusional compared to those with OCD [25].

4. Cognitive characteristics: individuals with BDD have difficulties in accurately identifying facial emotional expressions. They are more likely to identify expressions as anger compared to OCD or normal individuals [28]. Both BDD and OCD patients are preoccupied with body symmetrical appearance [25,29].

5. Psychiatric and personality comorbidities: social phobia, MDD, SUD, suicidal ideation are common in both disorders; but are more prevalent in patients with BDD. Patients with BDD have lifetime suicidal attempt rates of $22 \%$ compared to $8 \%$ in patients with OCD. People with OCD are more likely to have obsessivecompulsive personality disorder while those with BDD are more likely to have narcissistic, histrionic, and avoidant personality disorders [25].

Figure 1 explains the differences and the common features between OCD and BDD [25].

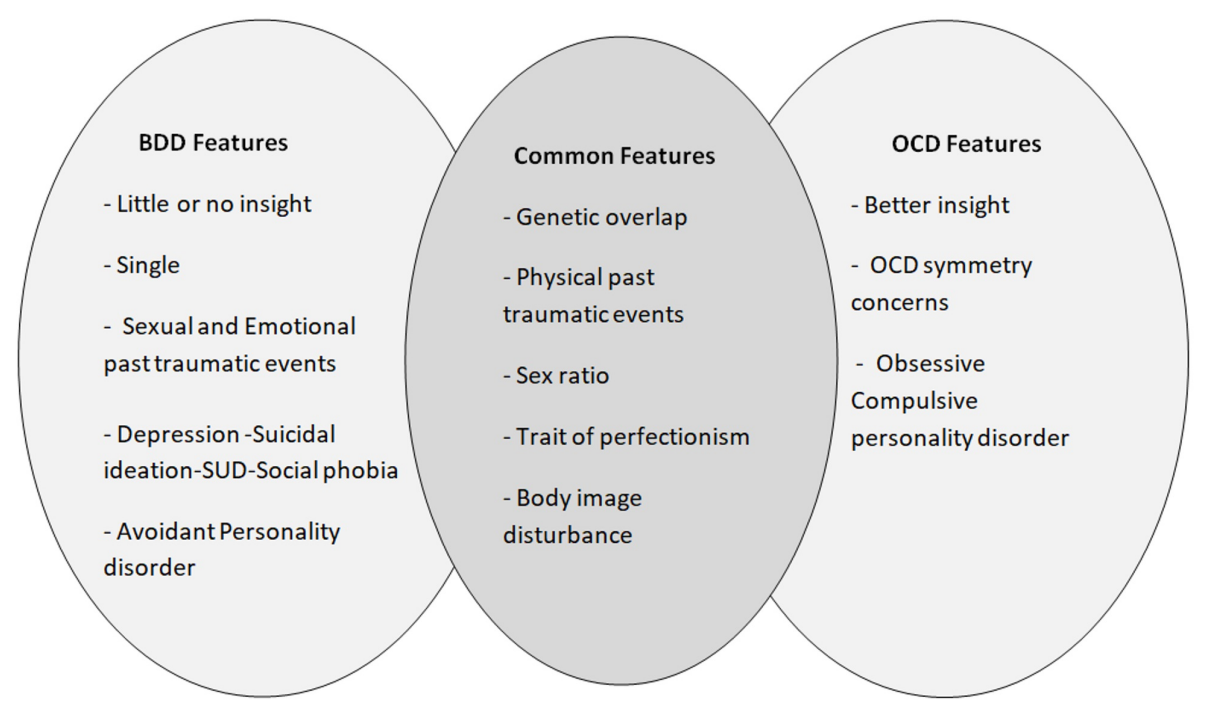

\section{FIGURE 1: The differences and the common features between OCD and BDD}

OCD: obsessive-compulsive disorder, BDD: body dysmorphic disorder, SUD: substance use disorder

Studies have found the prevalence of BDD in patients with OCD in a large patient sample was $8.7 \%$ to $15 \%$ compared to 3\% in non-OCD. Similarly, the prevalence of OCD in patients with BDD was $37 \%$ to $16.7 \%$ compared to $8 \%$ in non-BDD patients [25]. A cross-sectional study $(n=137)$ compared three groups of patients with BDD, OCD, and comorbid OCD-BDD. The study found patients with comorbid OCD-BDD have higher rates of bulimia, SUD, Bipolar II disorder, and social phobia [30]. A cross-sectional study $(\mathrm{n}=295)$ found people with comorbid OCD-BDD have high morbidity and poor psychosocial functions [31]. They also have higher rates of anxiety, schizotypal features, very poor insight, and suicidal ideation compared to those with BDD or OCD alone. The higher rates of comorbidities in the OCD-BDD group were the same as in the BDD group after controlling the symptom severity in BDD; however, it remained significant when compared with the OCD group [31]. The risk of comorbidity of OCD-BDD is three times higher in samples with a primary diagnosis of BDD compared to those with a primary diagnosis of OCD with $27.5 \%$ and $10.4 \%$, respectively [25]. 


\section{Study limitation}

Our study is based on reviewing research articles published after 1997 and does not include possible important contributions from studies published prior to that. A systematic review in our study was not performed and no quality assessment of the selected research studies was done.

\section{Conclusions}

OCD is a common psychiatric disorder associated with a high rate of psychiatric comorbidities and an increased risk of suicide. BDD is an underdiagnosed mental disorder that is commonly associated with depression, suicidal ideation, and attempts. The suicide risk in OCD is increased by the increase in the intensity of the obsessions, trait perfectionism and alexithymia. Individuals with BDD have poor insight and are usually single with past emotional and sexual traumatic events. The suicide risk in BDD is increased by the presence of comorbid SUD, MDD, eating disorders and personality disorders. OCD and BDD share common genetic and environmental risk factors. They also share common clinical features and sociodemographic profiles. Both OCD and BDD are related disorders that commonly coexist. People with comorbid OCD and BDD have a poor psychosocial outcome and are at high risk to develop social phobia, BD, SUD, and suicidal ideation. The suicide risk in OCD and BDD is mostly underestimated. Clinicians should be more aware of the various clinical presentations of BDD. It is recommended that dermatologists and cosmetic surgeons consult with mental health professionals for efficient diagnosis and proper referrals of patients with BDD. Patients with OCD should be properly screened for comorbid anxiety, depression, BDD, and suicidal ideation. Further original research studies are needed to investigate the relationship between OCD and BDD.

\section{Additional Information \\ Disclosures}

Conflicts of interest: In compliance with the ICMJE uniform disclosure form, all authors declare the following: Payment/services info: All authors have declared that no financial support was received from any organization for the submitted work. Financial relationships: All authors have declared that they have no financial relationships at present or within the previous three years with any organizations that might have an interest in the submitted work. Other relationships: All authors have declared that there are no other relationships or activities that could appear to have influenced the submitted work.

\section{References}

1. Ruscio AM, Stein DJ, Chiu WT, Kessler RC: The epidemiology of obsessive-compulsive disorder in the National Comorbidity Survey Replication. Mol Psychiatry. 2008, 15:53-63. 10.1038/mp.2008.94

2. Veale D, Singh A: Understanding and treating body dysmorphic disorder. Indian J Psychiatry. 2019, 61:131135. 10.4103/psychiatry.indianjpsychiatry_528_18

3. Veale D, Gledhill LJ, Christodoulou P, Hodsoll J: Body dysmorphic disorder in different settings: a systematic review and estimated weighted prevalence. Body Image. 2016, 18:168-186. 10.1016/j.bodyim.2016.07.003

4. Conroy M, Menard W, Fleming-Ives K, Modha P, Cerullo H, Phillips KA: Prevalence and clinical characteristics of body dysmorphic disorder in an adult inpatient setting. Gen Hosp Psychiatry. 2008, 30:6772. 10.1016/j.genhosppsych.2007.09.004

5. Phillips KA, Menard W, Fay C, Weisberg R: Demographic characteristics, phenomenology, comorbidity, and family history in 200 individuals with body dysmorphic disorder. Psychosomatics. 2005, 46:317-325. 10.1176/appi.psy.46.4.317

6. Fernández de la Cruz L, Rydell M, Runeson B, et al.: Suicide in obsessive-compulsive disorder: a populationbased study of 36788 Swedish patients. Mol Psychiatry. 2016, 22:1626-1632. 10.1038/mp.2016.115

7. Fenske JN, Pettersen K: Obsessive-compulsive disorder: diagnosis and management. Am Fam Physician. 2015, 92:896-903.

8. Angelakis I, Gooding PA, Panagioti M: Suicidality in body dysmorphic disorder (BDD): a systematic review with meta-analysis. Clin Psychol Rev. 2016, 49:55-66. 10.1016/j.cpr.2016.08.002

9. Williams MT, Mugno B, Franklin M, Faber S: Symptom dimensions in obsessive-compulsive disorder: phenomenology and treatment outcomes with exposure and ritual prevention. Psychopathology. 2013, 46:365-376. 10.1159/000348582

10. Rachamallu V, Song MM, Liu H, Giles CL, McMahon T: Obsessive-compulsive disorder with suicide obsessions in a first responder without previous diagnosis of OCD or history of suicide attempts. Case Rep Psychiatry. 2017, 2017:4808275. 10.1155/2017/4808275

11. Simpson HB, Slifstein M, Bender JJr, Xu X, Hackett E, Maher MJ, Abi-Dargham A: Serotonin 2A receptors in obsessive-compulsive disorder: a positron emission tomography study with [11C]MDL. Biol Psychiatry. 2011, 70:897-904. 10.1016/j.biopsych.2011.06.023

12. Pauls DL: The genetics of obsessive-compulsive disorder: a review . Dialogues Clin Neurosci. 2010, 12:149163.

13. Torres AR, Fontenelle LF, Shavitt RG, Ferrão YA, do Rosário MC, Storch EA, Miguel EC: Comorbidity variation in patients with obsessive-compulsive disorder according to symptom dimensions: results from a large multicentre clinical sample. J Affect Disord. 2016, 190:508-516. 10.1016/j.jad.2015.10.051

14. Pallanti S, Grassi G, Sarrecchia ED, Cantisani A, Pellegrini M: Obsessive-compulsive disorder comorbidity: clinical assessment and therapeutic implications. Front Psychiatry. 2011, 2:70. 10.3389/fpsyt.2011.00070

15. Albert U, De Ronchi D, Maina G, Pompili M: Suicide risk in obsessive-compulsive disorder and exploration of risk factors: a systematic review. Curr Neuropharmacol. 2019, 17:681-696. 
10.2174/1570159x16666180620155941

16. Kim H, Seo J, Namkoong K, Hwang EH, Sohn SY, Kim SJ, Kang JI: Alexithymia and perfectionism traits are associated with suicidal risk in patients with obsessive-compulsive disorder. J Affect Disord. 2016, 192:5055. 10.1016/j.jad.2015.12.018

17. Velloso P, Piccinato C, Ferrão Y, et al.: The suicidality continuum in a large sample of obsessive-compulsive disorder (OCD) patients. Eur Psychiatry. 2016, 38:1-7. 10.1016/j.eurpsy.2016.05.003

18. Krebs G, Fernández de la Cruz L, Mataix-Cols D: Recent advances in understanding and managing body dysmorphic disorder. Evid Based Mental Health. 2017, 20:71-75. 10.1136/eb-2017-102702

19. Phillips KA: Body dysmorphic disorder: recognizing and treating imagined ugliness. World Psychiatry. 2004, 3:12-17.

20. Ruffolo JS, Phillips KA, Menard W, Fay C, Weisberg RB: Comorbidity of body dysmorphic disorder and eating disorders: Severity of psychopathology and body image disturbance. Int J Eat Disord. 2006, 39:11-19. 10.1002/eat.20219

21. Grant JE, Phillips KA: Is anorexia nervosa a subtype of body dysmorphic disorder probably not, but read on . Harvard Rev Psychiatry. 2004, 12:123-126. 10.1080/10673220490447236

22. Pope CG, Pope HG, Menard W, Fay C, Olivardia R, Phillips KA: Clinical features of muscle dysmorphia among males with body dysmorphic disorder. Body Image. 2005, 2:395-400. 10.1016/j.bodyim.2005.09.001

23. Phillips KA: Suicidality in body dysmorphic disorder. Prim Psychiatry. 2007, 14:58-66.

24. Phillips KA, Didie ER, Menard W, Pagano ME, Fay C, Weisberg RB: Clinical features of body dysmorphic disorder in adolescents and adults. Psychiatry Res. 2006, 141:305-314. 10.1016/j.psychres.2005.09.014

25. Frías Á, Palma C, Farriols N, González L: Comorbidity between obsessive-compulsive disorder and body dysmorphic disorder: prevalence, explanatory theories, and clinical characterization. Neuropsychiatr Dis Treat. 2015, 11:2233-2244. 10.2147/ndt.s67636

26. Monzani B, Rijsdijk F, Iervolino AC, Anson M, Cherkas L, Mataix-Cols D: Evidence for a genetic overlap between body dysmorphic concerns and obsessive-compulsive symptoms in an adult female community twin sample. Am J Med Genet B Neuropsychiatr Genet. 2012, 159B:376-382. 10.1002/ajmg.b.32040

27. Phillips KA, Gunderson CG, Mallya G, McElroy SL, Carter W: A comparison study of body dysmorphic disorder and obsessive-compulsive disorder. J Clin Psychiatry. 1998, 59:568-575. 10.4088/jcp.v59n1102

28. Buhlmann U, Mcnally RJ, Etcoff NL, Tuschen-Caffier B, Wilhelm S: Emotion recognition deficits in body dysmorphic disorder. J Psychiatr Res. 2004, 38:201-206. 10.1016/s0022-3956(03)00107-9

29. Hart AS, Phillips KA: Symmetry concerns as a symptom of body dysmorphic disorder . J Obsessive Compuls Relat Disord. 2013, 2:292-298. 10.1016/j.jocrd.2013.04.004

30. Frare F, Perugi G, Ruffolo G, Toni C: Obsessive-compulsive disorder and body dysmorphic disorder: a comparison of clinical features. Eur Psychiatry. 2004, 19:292-298. 10.1016/j.eurpsy.2004.04.014

31. Phillips KA, Pinto A, Menard W, Eisen JL, Mancebo M, Rasmussen SA: Obsessive-compulsive disorder versus body dysmorphic disorder: a comparison study of two possibly related disorders. Depress Anxiety. 2007, 24:399-409. 10.1002/da.20232 